\title{
Invasão total de seio maxilar por ceratocisto odontogênico
}

\author{
Total maxillary sinus invasion by odontogenic keratocyst \\ Invasión total del seno maxilar por queratoquiste odontogénico
}

Recebido: 02/06/2021 | Revisado: 08/06/2021 | Aceito: 09/06/2021 | Publicado: 23/06/2021

\author{
Aída Juliane Ferreira dos Santos \\ ORCID: https://orcid.org/0000-0001-8055-3120 \\ Universidade de Pernambuco, Brasil \\ E-mail: aida.juliane@upe.br \\ Carolina Chaves Gama Aires \\ ORCID: https://orcid.org/0000-0001-9251-2895 \\ Universidade de Pernambuco, Brasil \\ E-mail: carolina.aires@upe.br \\ Miquéias Oliveira de Lima Júnior \\ ORCID: https://orcid.org/0000-0001-8854-1132 \\ Universidade de Pernambuco, Brasil \\ E-mail: miqueias_lima_junior@hotmail.com \\ Thiago Coelho Gomes da Silva \\ ORCID: https://orcid.org/0000-0003-1750-8372 \\ Universidade de Pernambuco, Brasil \\ E-mail: thiagocoelhogs@gmail.com \\ Belmiro Cavalcanti do Egito Vasconcelos \\ ORCID: https://orcid.org/0000-0002-6515-1489 \\ Universidade de Pernambuco, Brasil \\ E-mail: belmirovasconcelos@gmail.com
}

\begin{abstract}
Resumo
Introdução: O ceratocisto odontogênico é um tumor benigno acometido em sua maioria em paciente do sexo masculino com menos de 30 anos. Deriva-se da lâmina dentária, de seus restos ou dos primórdios de um dente normal ou supranumerário, antes da formação dos tecidos calcificados. Equivale a $11 \%$ de todos os cistos odontogênicos, em que $1 \%$ deles acometem o seio maxilar. Clinicamente nas lesões maiores, podem apresentar dor, tumefação e drenagem, onde seu diagnóstico é confirmado através do exame de imagem em conjunto com histopatológico. Objetivo: Relatar um caso de ceratocisto odontogênico grande em maxila, o qual invadiu o seio maxilar esquerdo desde o osso alveolar até o nervo infraorbital, em um paciente com 51 anos de idade. Discussão: Dentro dos tratamentos mais agressivos temos as menores taxas de recidiva de lesão, porém têm desvantagens bem significativas que vão desde neuropatias até desfiguração do paciente, desta forma, em cistos grandes os tratamentos mais conservadores devem ser priorizados. Conclusão: Inicialmente devem ser escolhidos os tratamentos que reduzem a agressividade e o volume cístico tais como descompressão ou marsupialização, como uma forma de proteção às estruturas nobres como o nervo alveolar inferior e o infraorbital e concomitantemente propiciar um segundo tempo cirúrgico com uma lesão menor e menos agressiva. Palavras-chave: Seio maxilar; Cisto odontogênico; Tumor odontogênico.
\end{abstract}

\begin{abstract}
Introduction: The odontogenic keratocyst is a cystic lesion that is derived from the dental lamina, its remains or the beginnings of a normal or supernumerary tooth, before the formation of calcified tissues. It is equivalent to $11 \%$ of all odontogenic cysts, and only $1 \%$ of them affect the maxillary sinus. Clinically, in larger lesions, they may present with pain, swelling and drainage, where their diagnosis is confirmed by imaging together with histopathology. Objective: To report a case of a large odontogenic keratocyst in the maxilla, which invaded the left maxillary sinus from the alveolar bone to the infraorbital nerve, in a 51-year-old patient. Discussion: Among the most aggressive treatments we have the lowest lesion recurrence rates, but they have very significant disadvantages ranging from neuropathies to patient disfigurement, therefore, in large cysts, the most conservative treatments should be prioritized. Conclusion: Initially, treatments that reduce aggression and cystic volume should be chosen, such as decompression or marsupialization, as a way of protecting noble structures such as the lower alveolar nerve and the infraorbital nerve, and at the same time provide a second surgical time with a minor injury and less aggressive.
\end{abstract}

Keywords: Maxillary sinus; Odontogenic cysts; Jaw cysts.

\section{Resumen}

Introducción: El queratoquiste odontogénico es una lesión quística que se deriva de la lámina dental, sus restos o los inicios de un diente normal o supernumerario, antes de la formación de tejidos calcificados. Equivale al $11 \%$ de todos los quistes odontogénicos, y solo el $1 \%$ de ellos afecta al seno maxilar. Clínicamente, en lesiones de mayor tamaño, 
pueden presentarse con dolor, hinchazón y supuración, donde su diagnóstico se confirma mediante imagen junto con histopatología. Objetivo: Presentar un caso de queratoquiste odontogénico de gran tamaño en el maxilar, que invadió el seno maxilar izquierdo desde el hueso alveolar hasta el nervio infraorbitario, en un paciente de 51 años. Discusión: Dentro de los tratamientos más agresivos, tenemos las tasas más bajas de recurrencia de lesiones, pero tienen desventajas muy importantes, que van desde neuropatías hasta desfiguración del paciente, por lo que, en quistes grandes, se deben priorizar los tratamientos más conservadores. Conclusión: Inicialmente se deben optar por tratamientos que reduzcan la agresión y el volumen quístico, como la descompresión o la marsupialización, como forma de proteger estructuras nobles como el nervio alveolar inferior y el nervio infraorbitario, y al mismo tiempo proporcionar un segundo tiempo quirúrgico con una lesión menor y menos agresiva.

Palabras clave: Seno maxilar; Quistes odontogénicos; Quistes maxilomandibulares.

\section{Introdução}

Considerado um tumor odontogênico entre 2005 e 2017 devido ao seu comportamento agressivo, atualmente, o ceratocisto é classificado como um cisto de origem odontogênica. O ceratocisto é um cisto caracterizado por uma fina camada de epitélio escamoso estratificado paraqueratinizado com células basais hipercromáticas em paliçada A respeito do seu desenvolvimento, a teoria mais aceita seria por advir de restos celulares da lâmina dentária, apesar de outra hipótese sugerir a origem da lesão a partir da proliferação de células da camada basal do epitélio oral da mandíbula ou da maxila (Mallmann et al., 2012; Moura et al., 2016). De todos os cistos de origem odontogênica, $11 \%$ são diagnosticados como ceratocisto odontogênico (CO), sendo terceiro cisto mais comum nos maxilares. Ocorre em uma ampla faixa etária, com picos de incidência na segunda e terceira décadas de vida podendo variar da infância até a velhice, entretanto $60 \%$ dos casos são vistos em pessoas entre 10 e 40 anos de idade (IARC, 2017).

As áreas anatômicas frequentemente envolvidas são as regiões do corpo, ramo ascendente e do ângulo da mandíbula, afetando menos frequentemente, a maxila (Al-Moraissi et al., 2016). Segundo Neville et al (2016), esta região é afetada em apenas $13 \%$ dos casos e as lesões mais extensas chegam a comprometer o seio maxilar. Cistos na região posterior da maxila, bem como cistos múltiplos, são mais associados com a síndrome do carcinoma nevóide basocelular. Essa síndrome é caracterizada pela presença de múltiplos ceratocistos nos maxilares, junto com carcinomas basocelulares, anomalias das costelas, calcificação intracraniana, entre outras características (IARC, 2017).

Os CO’s menores, comumente são assintomáticos, sendo descobertos somente durante exames de imagem de rotina. Os maiores podem causar dor, tumefação ou apresentar drenagem. Geralmente a lesão possui uma cápsula fibrosa, fina e friável, onde desta forma o epitélio fica fracamente ligado a ela, dificultando a remoção de todo o revestimento epitelial (Bagheri, 2013). Devido a isso, o CO é uma das lesões de origem odontogênica mais agressivas, com alto índice de recorrência e tendência a invadir tecidos adjacentes. A taxa de recorrência pode depender do tamanho da lesão, a modalidade de tratamento empregada para a erradicação da lesão e invasão de células-filhas nos tecidos circundantes (Morankar et al., 2018).

Vários fatores devem ser levados em consideração na tomada de decisões, dentre as opções terapêuticas incluindo idade do paciente, tamanho e localização do cisto, envolvimento dos tecidos moles, história de tratamento e a variante histológica da lesão. As abordagens cirúrgicas objetivam a remoção ou diminuição da lesão, seguida ou não de tratamentos adjuvantes da cavidade óssea remanescente. Em alguns casos, a ressecção marginal é necessária (Khan et al., 2019; Morankar et al., 2018).

Nesse contexto, o objetivo do presente trabalho é relatar o caso clínico, bem como discutir a abordagem terapêutica utilizada, de um ceratocisto odontogênico em maxila de grandes proporções que se estendeu desde a região alveolar maxilar, infiltrando-se pelo seio maxilar até o assoalho de órbita, um acontecimento de cunho raro por sua localização e tamanho.

\section{Metodologia}

Este artigo é um estudo de caso único, de caráter qualitativo e descritivo. De acordo com Pereira et al. (2018), pesquisas desta natureza caracterizam-se por elucidar um determinado assunto e estuda-lo minuciosamente, através do acesso aos registros 
médicos, exame clínico, exames laboratoriais e de imagem fornecidos, sendo o pesquisador o instrumento primordial. Foi consultada a literatura em bancos de dados como PubMed e LILACS, realizando uma análise dos artigos relevantes ao tema do estudo. Foram selecionados 25 artigos para a construção do artigo.

\section{Relato de Caso}

Paciente do sexo masculino, 50 anos, feoderma, compareceu a um serviço de cirurgia e traumatologia buco maxilo facial de um hospital público do Recife, com relato de aumento de volume em região maxilar superior esquerda percebido há dois meses. Durante exame físico intraoral, observou-se uma tumefação maxilar do lado esquerdo indolor, apagamento do sulco nasolabial ipsilateral, sem saída de secreção local. Após exame clínico inicial, solicitouse uma tomografia de face que apresentou uma imagem hipodensa extensa com limites definidos e sugestiva de lesão cística, estendendo-se da região amelo-cementária dos elementos dentários até o assoalho de órbita, ocasionando expansão do seio maxilar com pontos de rarefação óssea nas regiões de abertura piriforme e pterigomaxilar.

Durante análise inicial, realizou-se uma punção aspirativa com saída de líquido amarelo citrino gerando a hipótese diagnóstica de ceratocisto odontogênico. Foi planejado e executado um procedimento sob anestesia local para instalação de dispositivo de descompressão localizado acima dos pré-molares esquerdos (Figura 1), e uma biópsia incisional, removendo material cístico para análise histopatológica.

Figura 1 - Aspecto clínico do dispositivo já instalado.

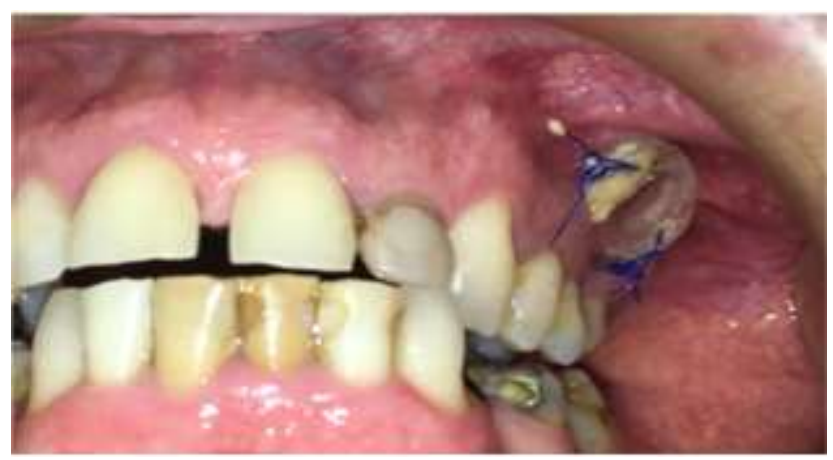

Fonte: Autores (2021).

O paciente foi orientado a irrigar 2-3 vezes ao dia, com clorexidina $0,12 \%$ e soro fisiológico $0,9 \%$. O exame histopatológico da lesão evidenciou um tecido epitelial pavimentoso com paraqueratina indicativo de ceratocisto odontogênico. Após quatro meses de descompressão foi observado extensa regressão da lesão e normalização do aspecto extra e intraoral do paciente, com contornos ósseos quase regularizados (Figura 2). 
Figura 2 - Aspecto tomográfico antes (A) e depois da descompressão (B).

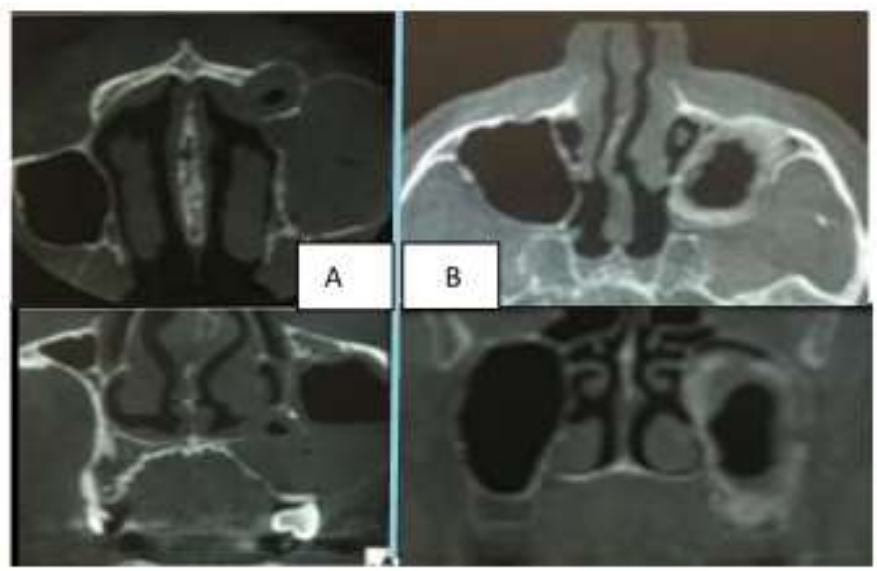

Fonte: Autores (2021).

Após a redução da lesão, seguiu-se com a enucleação e curetagem cística sob anestesia geral. O procedimento iniciouse conforme rotina cirúrgica, com a anti-sepsia intra e extraoral, aposição dos campos operatório e anestesia local para hemostasia. O dispositivo descompressivo foi removido e realizado um acesso cirúrgico com descolamento do retalho mucoperiosteal. A cortical óssea vestibular encontrava-se extremamente fragilizada, sendo posteriormente removida. Realizouse a exodontia dos elementos dentários 23, 24 e 25 associados à lesão por já possuírem um grau avançado de mobilidade. A enucleação e curetagem cística foi associada a uma osteotomia periférica em região alveolar e sinusal (Figura 3), no qual observou-se a exposição do nervo infraorbital e seu íntimo contato com a cápsula cística.

Figura 3 - Momento trans cirúrgico após a exodontia e osteotomia periférica.

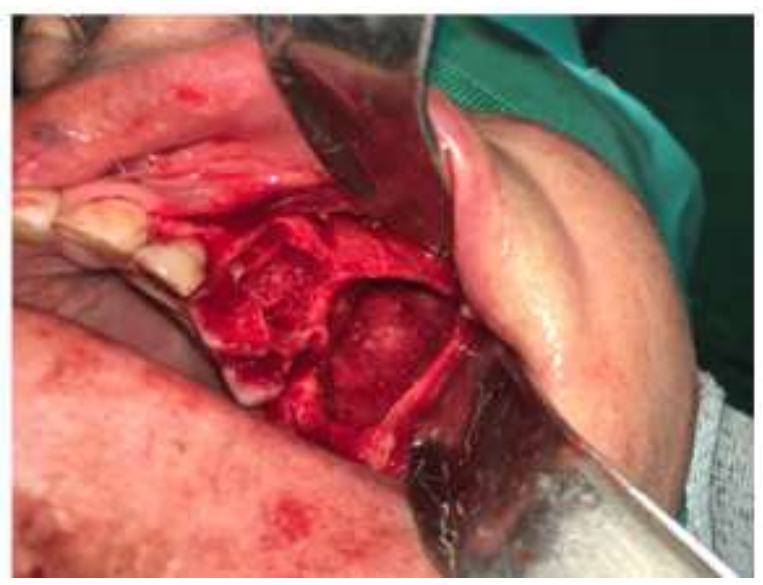

Fonte: Autores (2021).

Posteriormente, realizou-se a regularização óssea, toalete da cavidade óssea com soro fisiológico 0,9\%, reposição passiva do retalho mucoso e sutura interpapilar com fio reabsorvível Catgut 4.0 (Figura 4). O material colhido foi novamente levado à análise histopatológica, corroborando a hipótese diagnóstica inicial de ceratocisto odontogênico. Não foram relatadas complicações pós-operatórias. O paciente encontra-se em acompanhamento há cinco anos, sem ocorrência de recidiva da lesão até o momento. 
Figura 4 - Sutura passiva realizada com Catgut 4.0.

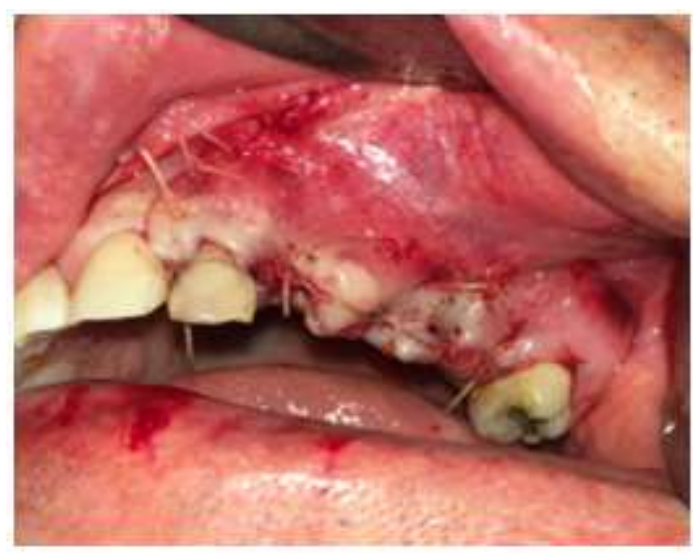

Fonte: Autores (2021).

\section{Discussão}

O presente estudo relatou o caso de um paciente do sexo masculino na quinta década de vida apresentando um ceratocisto odontogênico em região posterior de maxila envolvendo o seio maxilar, uma ocorrência clínica rara devido a faixa etária e localização da lesão. A maioria dos pacientes diagnosticados tem menos de 30 anos, existindo uma menor incidência nos pacientes entre a quinta e a sétima década de vida (IARC, 2017). A associação dessa lesão cística com dentes irrompidos, que ocorre em 30\% dos casos (Sánchez-Burgos, et al., 2014; MacDonald, 2016), não foi encontrada neste caso.

Quando na mandíbula, o ceratocisto tem tendência a crescer predominantemente no sentido mésio-distal, ao longo do comprimento do osso, causando expansão mínima das placas corticais vestibular e lingual (Mosier, 2015). Em contraste, grandes cistos na maxila apresentam mais frequentemente uma expansão do osso alveolar com remodelação, afinamento, recorte e perfuração das corticais (Koenig, et al., 2017). A diferença entre o padrão de crescimento destes cistos mandibulares e maxilares pode ser explicada parcialmente devido à maior espessura cortical da mandíbula em comparação com a da maxila (MacDonald, 2016). A sua taxa de crescimento geralmente é menor que a de outros cistos maxilares, mas ocorre de forma mais constante, com estudos mostrando que parecem seguir o caminho da menor resistência. Aproximadamente $65 \%$ deles ocorrem em mandíbula, tendo predileção pela região posterior. Em maxilas, são mais raros envolvendo cerca de 13\% (Neville, 2016). O envolvimento do seio maxilar, como ocorrido no presente caso, é mais raro ainda, ocorrendo em menos de 1\% dos casos (Press, 2008).

Os CO’’s são lesões clinicamente agressivas, e sua maior particularidade é a taxa de recorrência elevada, possivelmente ligada aos restos de epitélio cístico e potencial intrínseco de crescimento após a excisão (Santos, 2005), podendo ocorrer em até $62 \%$ dos seus casos (Takata, 2005). Durante o manejo das lesões de curso clínico mais agressivo, tais quais o ceratocisto, sabese que a descompressão e/ou marsupialização leva a uma redução significativa no tamanho e espessamento do revestimento da lesão, seguida de enucleação secundária, preservando assim as estruturas vitais associadas (Morankar et al., 2018). Atualmente, não existe consenso sobre o melhor manejo desta lesão. Tanto a descompressão quanto a marsupialização tem a mesma função, o que as difere é a instalação de um dispositivo para manutenção da abertura cirúrgica no caso da descompressão, enquanto na marsupialização a janela cirúrgica é suturada junto à mucosa bucal. Ambas as técnicas foram consideradas eficazes como um tratamento preliminar para grandes ceratocistos (Nakamura et al., 2002). Já foi observado que há mudanças histológicas no epitélio depois dessas técnicas, sendo recomendadas para grandes cistos por torná-los potencialmente menores e menos agressivos (Myoung et al., 2001). Todavia quando de forma isolada, nem sempre são eficientes e uma possível causa disso é devido à presença de cistos satélites, que permanecem mesmo após a intervenção (Pinto et al., 2016). 
Esta alta taxa de recorrência está normalmente relacionada a uma parede cística delgada e friável, tornando difícil a completa enucleação da lesão em uma única peça. Para isso, faz-se necessário o tratamento adjuvante da cavidade óssea remanescente. Essas terapias complementares visam o tratamento de uma camada de 1 a $2 \mathrm{~mm}$ de osso em torno da cavidade cística, a fim de se evitar recidivas da lesão por remanescentes celulares císticos, através da osteotomia periférica, do uso da solução de Carnoy ou a crioterapia com nitrogênio líquido (Al-Moraissi et al., 2016; Hauer et al., 2020).

A solução de Carnoy consiste em um agente de cauterização química com rápida fixação local e ação hemostática para remover possíveis cistos satélites, porém deve-se ter atenção para não ser aplicada diretamente sobre tecidos nervosos, pois isso poderia acarretar danos como parestesia, sensação de queimação ou perda total da sensibilidade a depender do tempo de aplicação, onde se recomenda a utilização por um tempo limite de três minutos para preservação do feixe nervoso. Além disso, como o CO é mais comum na posterior de mandíbula, a literatura foca em estudar a aplicação na solução de Carnoy no nervo alveolar inferior, não possuindo estudos suficientes com o nervo infraorbital (Gao et al., 2018).

O nitrogênio líquido por sua vez, apresenta a capacidade de eliminar o componente orgânico no local da lesão até 1,5 $\mathrm{mm}$ de profundidade, mantendo intacta a arquitetura inorgânica do osso (Ribeiro-Júnior et al., 2017), o que é apropriado para o manejo do ceratocisto, porém pode gerar necrose óssea local e não pode ser utilizado se tiver perfuração nas corticais ósseas por produzir necrose também nos tecidos moles adjacentes. Além disso, Bagheri (2013) contra indica a aplicação da solução de Carnoy e o nitrogênio líquido em seios maxilares. No referido caso, após a realização da descompressão por 4 meses, o paciente foi submetido a uma enucleação secundária seguida de uma osteotomia periférica da cavidade óssea, visando eliminar possíveis restos epiteliais patológicos na cavidade óssea remanescente. Preferiu-se a osteotomia periférica devido à proximidade com o nervo infraorbitário e a localização da lesão, minimizando o risco de parestesia e de necrose dos tecidos adjacentes.

Em tratamentos mais agressivos, tais quais as ressecções cirúrgicas, são encontradas taxas menores de recorrência, chegando a 0\% em casos tratados por ressecção (Blanas,2002). No entanto, essa abordagem tem muitas desvantagens que devem ser consideradas, como a desfiguração facial. Desta forma, a ressecção é reservada para lesões que se caracterizam como muito agressivas e destrutivas, que já se realizou várias cirurgias conservadoras sem sucesso ou onde a lesão não está mais contida apenas ao osso, existindo envolvimento de tecidos moles com potencial envolvimento de estruturas vitais (Zhao et al., 2002). Por essas razões, os tratamentos cirúrgicos mais conservadores devem ser priorizados sempre que possível (Ribeiro-Júnior et al., 2017).

\section{Considerações Finais}

Vários tratamentos propostos para os ceratocistos odontogênicos são discutidos na literatura, bem como a necessidade ou não de abordagens complementares. A escolha do tratamento está subordinada a diversos fatores, dentre eles a idade do paciente, a taxa de recorrência, a natureza agressiva e invasiva da lesão, o local, o tamanho e sua relação com estruturas anatômicas importantes. A partir disso, pode-se avaliar que tipos de abordagens estão indicadas, sendo conservadoras ou radicais, visando a manutenção de estruturas importantes, a redução da lesão e diminuir os riscos de recidivas.

Pela raridade do acontecimento de um $\mathrm{CO}$ acometendo o seio maxilar por completo, existe uma escassez de estudos que abordem tratamentos especifico para área, visto que o seio possui estruturas anatômicas complexas como o nervo infraorbital, nervo alveolar superior, ramos da artéria maxilar e facial, dentre outros, existindo neste assunto uma necessidade de estudos futuros. 


\section{Referências}

Al-Moraissi, E. A., Pogrel, M. A., \& Ellis, E. (2016). Enucleation with or without adjuvant therapy versus marsupialization with or without secondary enucleation in the treatment of keratocystic odontogenic tumors: A systematic review and meta-analysis. Journal of Cranio-Maxillofacial Surgery, 44(9), 1395-1403. https://doi.org/10.1016/j.jcms.2016.05.020

Blanas, N., Freund, B., Schwartz, M., \& Furst, I. M. (2000). Systematic review of the treatment and prognosis of the odontogenic keratocyst. Oral Surgery, Oral Medicine, Oral Pathology, and Oral Radiology Endodontology. 90(5):553-8. 10.1067/moe.2000.110814

Bagheri, S. C., Bell, R. B., Khan, H. A. (2013). Terapias Atuais em Cirurgia Bucomaxilofacial. Elsevier.

Gao, L., Ren, W., Li, S., Zheng, J., Xue, L., Xu, Y., Wang, Q., Song, J., Dou, Z., Zhou, M., Xiao, W., \& Zhi, K. (2018). CBCT-based bone quality assessment in decompression of large odontogenic cystic lesions. Oral Radiology, 34(3), 251-256. https://doi.org/10.1007/s11282-018-0320-5

Hauer, L., Seidlová, P., Merglová, V., Hrusak, D., Böhmová, H., Posta, P., Gencur, J., \& Netolicky, J. (2020). Complete removal of dentigerous cysts with preservation of associated teeth as an alternative to marsupialization in children and preadolescents. Journal of Cranio-Maxillofacial Surgery, 48(8), 808-814. https://doi.org/10.1016/j.jcms.2020.05.014

International agency for research on cancer (IARC). (2017). WHO Classification of head and neck tumours. Lyon: IARC.

Koenig, L. J., Tamimi, D. F., Petrikowski, C. G., \& Perschbacher, S. E. (2017). Diagnóstico por imagem: oral e maxilofacial, Elsevier

Khan, A. A., Qahtani, S. Al, Dawasaz, A. A., Saquib, S. A., Asif, S. M., Ishfaq, M., Kota, M. Z., \& Ibrahim, M. (2019). Management of an extensive odontogenic keratocyst: A rare case report with 10-year follow-up. Medicine (United States), 98(51). https://doi.org/10.1097/MD.0000000000017987

MacDonald D (2016) Lesões dos maxilares apresentando-se como radiolucências na TC de feixe cônico. Clin Radiol 71: $972-985$

Mallmann, C.T. Vieira, R. R., Silva, S. O., Carli, B. M. G. \& Carli, J. P. (2012)Tumor odontogênico ceratocístico: levantamento de casos e revisão de literatura. Revista do curso de Odontologia da Faculdade da Saúde da Universidade Metodista de São Paulo - UMESP. $20,67-72$.

Morankar, R., Bhatia, S. K., Goyal, A., \& Gulia, P. (2018). Conservative management of keratocystic odontogenic tumour in a young child with decompression and an intraoral appliance: 5-year follow-up. BMJ Case Reports, 2018. https://doi.org/10.1136/bcr-2017-221563.

Mosier, K.M. (2015) Lesões da mandíbula. Semin Ultrasound CT MR 36: 444-450.

Moura, B. S., Cavalcante, M. A., \& Hespanhol, W. (2016)Tumor odontogênico ceratocístico, Revista do Colégio Brasileiro de. Cirurgiões, 43(6), 466-471.

Myoung, H., Hong, S. P., Hong, S. D., Lee, J. Il, Lim, C. Y., Choung, P. H., Lee, J. H., Choi, J. Y., Seo, B. M., \& Kim, M. J. (2001). Odontogenic keratocyst: Review of 256 cases for recurrence and clinicopathologic parameters. Oral Surgery, Oral Medicine, Oral Pathology, Oral Radiology, and Endodontics, 91(3), 328-333. https://doi.org/10.1067/moe.2001.113109

Nakamura, N., Mitsuyasu, T., Mitsuyasu, Y., Taketomi, T., Higuchi, Y., \& Ohishi, M. (2002). Marsupialization for odontogenic keratocysts: Long-term followup analysis of the effects and changes in growth characteristics. Oral Surgery, Oral Medicine, Oral Pathology, Oral Radiology, and Endodontics, 94(5), 543553. https://doi.org/10.1067/moe.2002.128022

Neville, B.W., Damm, D.D., Allen, C.M., \& Bouquot, J.E. Patologia Oral e Maxilofacial. Trad. Elsevier, 2009, 972p. Pathol Oral Radiol Endod. 2001; 91: 32833.

Pereira, A., Shitsuka, D., Parreira, F., \& Shitsuka, R. (2018). Metodologia da pesquisa científica. UFSM. https://repositorio.ufsm.br/bitstream/handle/1/19110/C urso_Lic-Ed-Esp_Did\%C3\%A1 tica-Geral.pdf?sequence=1\&isAllowed=y

Pinto, G. N. de S., Figueira, J. A., Gonçales, E. S., Sant’ana, E., \& Tolentino, E. D. S. (2016). Marsupialização como tratamento definitivo de cistos odontogênicos: relato de dois casos. Revista Da Faculdade de Odontologia - UPF, 20(3), 361-366. https://doi.org/10.5335/rfo.v20i3.5209

Press, S. G. (2008). Odontogenic tumors of the maxillary sinus. In Current Opinion in Otolaryngology and Head and Neck Surgery (Vol. 16, Issue 1, pp. 4754). https://doi.org/10.1097/MOO.0b013e3282f419da

Ribeiro-Júnior, O., Borba, A. M., Alves, C. A. F., Gouveia, M. M. de, Deboni, M. C. Z., \& Naclério-Homem, M. da G. (2017). Reclassification and treatment of odontogenic keratocysts: A cohort study. Brazilian Oral Research, 31, e98. https://doi.org/10.1590/1807-3107bor-2017.vol31.0098

Santos, T.S., Antunes, A.A., Avelar, R.L., Antunes, A.P. Cistos odontogênicos: estudo epidemiologico de 72 casos. Revista Brasileira de Cirurgiões Cabeça e Pescoço 2005; 36(1):30-32.

Sánchez-Burgos, R., González-Martín-Moro, J., Pérez-Fernández, E., \& Burgueño-García, M. (2014). Clinical, radiological and therapeutic features of keratocystic odontogenic tumours: A study over a decade. Journal of Clinical and Experimental Dentistry, 6(3), e259. https://doi.org/10.4317/jced.51408

Takata, T., \& Slootweg, P. (2005) Calcifying epithelial odontogenic tumour. In: World Health Organization. Classification of Tumors: head and neck tumours. Lyon: WHO. p.302-3.

Zhao, Y. F., Wei, J. X., \& Wang, S. P. (2002). Treatment of odontogenic keratocysts: A follow-up of 255 Chinese patients. Oral Surgery, Oral Medicine, Oral Pathology, Oral Radiology, and Endodontics, 94(2), 151-156. https://doi.org/10.1067/moe.2001.125694 